\title{
Multiple primary melanomas: Our experience
}

\author{
LOREDANA UNGUREANU ${ }^{1 *}$, IULIA ZBORAS ${ }^{1 *}$, ALINA VASILOVICI $^{1}$, ȘTEFAN VESA $^{2}$, \\ IOANA COSGAREA ${ }^{3}$, RODICA COSGAREA ${ }^{1}$ and SIMONA ȘENILA ${ }^{1}$
}

\author{
${ }^{1}$ Department of Dermatology, 'Iuliu Hațieganu' University of Medicine and Pharmacy, 400006 Cluj-Napoca; \\ ${ }^{2}$ Department of Pharmacology, 'Iuliu Hațieganu' University of Medicine and Pharmacy, \\ Toxicology and Clinical Pharmacology, 400012 Cluj-Napoca, Romania; ${ }^{3}$ Institute of Cellular Medicine, \\ University of Newcastle, Newcastle upon Tyne NE2 4HH, UK
}

Received September 2, 2020; Accepted October 2, 2020

DOI: $10.3892 / \mathrm{etm} .2020 .9520$

\begin{abstract}
Patients with melanoma have an increased risk of having other neoplasms, and particularly other melanomas and non-melanoma skin cancers. The study aimed to describe multiple primary melanomas in a large medical university centre from Romania (Cluj-Napoca) from 2004 to 2020. Out of 699 patients with melanoma included in the study, 26 (3.71\%) developed multiple tumours. The 26 patients developed a total of 59 melanomas, corresponding to a mean of 2.3 melanomas per patient. The site and histological subtype of the first and second melanomas were not consistent. The proportion of subsequent melanomas that were in situ $(51.5 \%)$ or thin melanomas ( $<1 \mathrm{~mm}, 24.2 \%)$ was higher compared with first melanomas $(7.7 \%$, respectively $11.5 \%)$. The median and mean time to diagnosis was 2.75 months, respectively, 28.09 months. In total, $76.92 \%$ of second melanomas were detected within three years, but we were able to document a subsequent melanoma more than ten years after the first diagnosis. The study highlights the importance of follow-up in patients diagnosed with melanoma, not only in the first years after the primary diagnoses but for the entire life.
\end{abstract}

\section{Introduction}

Although cutaneous melanoma represents only $10 \%$ of the total cutaneous malignant tumours, it is responsible for over $90 \%$ of the deaths caused by these tumours (1). Incidence rates of cutaneous melanoma are increasing worldwide in the

Correspondence to: Dr Loredana Ungureanu, Department of Dermatology, 'Iuliu Hațieganu' University of Medicine and Pharmacy, 3-6 Clinicilor Street, 400006 Cluj-Napoca, Romania E-mail: danaszent@yahoo.com

${ }^{*}$ Contributed equally

Key words: multiple primary melanomas, second melanoma, subsequent melanoma, synchronous melanoma, asynchronous melanomas fair-skinned population (2). However, early detection and the development of new drugs for metastatic melanoma lead to an increased survival rate and, therefore, an increasing population at risk of developing other neoplasms, especially cutaneous malignant tumours (3-5). It is well known that patients with melanoma have an increased risk of having other neoplasms, and particularly other melanomas and non-melanoma skin cancers (6-16). A personal history of melanoma proved to be a potent risk factor for the development of a subsequent primary melanoma (6-16). Multiple lesions can be detected as synchronous or asynchronous lesions. Synchronous lesions are defined as subsequent primary melanomas diagnosed within three months of the incident primary melanoma (7). Studies show that the percentage of patients who develop multiple primaries ranges from 0.2 to $8.6 \%$, out of which $26-40 \%$ develop as synchronous lesions $(7,8)$. The risk of a subsequent primary melanoma seems to be higher in the first year following the first diagnosis, but it remains increased for at least 20 years $(7,8)$.

The present study aimed to describe multiple primary melanomas in a large medical university centre from Romania (Cluj-Napoca) from 2004 to 2020, focusing on the number of lesions detected, tumour and patient characteristics, as well as time to subsequent diagnosis.

\section{Materials and methods}

Setting. This observational, retrospective, cohort study was carried out in Cluj County, in the North-Western Region of Romania. The present study was approved by the Ethics Committee of 'Iuliu Hatieganu' University of Medicine and Pharmacy (Cluj-Napoca, Romania).

Participants. All patients diagnosed with melanoma and followed-up in the Dermatology Department of the Cluj-Napoca Emergency County Hospital were eligible for analysis. Patients identified as having more than one invasive or in situ melanoma, followed up for at least one year, were included in the study. Data on patient characteristics included age, sex, family history of melanoma, presence of atypical nevi. The tumour was characterised according to the site (head and neck, trunk, upper limbs and lower limbs), histological subtype [superficial spreading melanoma (SSM), lentigo 
maligna melanoma (LMM), nodular melanoma (NM) and acral lentiginous melanoma (ALM) other], other histological characteristics (Breslow index, presence of mitosis, ulceration, vascular and neural invasion) and melanoma stage. The time to diagnosis was defined as the time from the first melanoma diagnosis to subsequent melanoma diagnosis. Subsequent melanomas were divided into synchronous and asynchronous melanomas. Synchronous melanomas were defined as those diagnosed simultaneously or within the first three months after the diagnosis of the first melanoma.

Statistical analysis was carried out using the MedCalc Statistical Software version 19.2.1 (MedCalc Software Ltd.; https://www.medcalc.org; 2020). Quantitative variables were expressed as mean and standard deviation, or median and 25-75 percentiles, depending on the normality of distribution. Qualitative data were characterised as frequency and percentage. Comparisons between groups were performed with Man-Whitney or chi-square test. The agreement between several variables (location and histology of melanoma) was established with Cohen's kappa coefficient. A P-value $<0.05$ was considered statistically significant.

\section{Results}

During the 16 years of the study period, 699 patients developed 732 melanomas. Out of the study population, 318 (45.4\%) were men, and $381(54.5 \%)$ were women. Of all patients, $673(96.28 \%)$ developed only one primary melanoma, whereas $26(3.71 \%), 11$ men and 15 women, developed multiple tumours. The median age of the patients having multiple primaries at the diagnosis of the first melanoma was 55.34 (25-75th percentile: 40.37-64.29) and the mean 52.47 years (SD 14.129). There were no statistically significant differences regarding gender and age between patients with one or multiple primary melanomas.

The 26 patients with multiple primaries developed a total of 59 melanomas (26 for men and 33 for women), corresponding to a mean of 2.3 melanomas per patient. Most patients $(n=21$, $80.76 \%$ ) developed only 2 melanomas; 3 patients $(11.53 \%)$ developed 3 melanomas and 2 patients (7.69\%) developed 4 melanomas.

Table I describes the tumour characteristics of the first melanoma, compared with the characteristics of subsequent melanomas.

We studied the concordance between the site of the first and second melanoma, and we found a fair concordance $(\mathrm{P}=0.007)$ (Table II). In 14 of the second melanomas $(53.84 \%)$ the site coincided with the first melanoma, a fact that occurred more frequently on melanomas occurring on the head and neck $(80 \%)$, followed by those on the trunk $(61.5 \%)$.

Regarding the histological subtype, the superficial spreading melanoma was the most frequent type in both first and subsequent melanomas, but nodular melanoma was more frequently seen as the first primary. We studied the concordance between the histological subtype of the first and second melanoma, but we found no concordance $(\mathrm{P}=0.087)$ (Table III).

Analysis of the distribution of multiple primary melanomas with respect to their Breslow index, showed that a higher proportion of subsequent melanomas were in situ $(51.5 \%)$ or thin melanomas $(<1 \mathrm{~mm}, 24.2 \%)$ compared with first melanomas $(7.7 \%$, respectively $11.5 \%)$, the difference being statistically significant $(\mathrm{P}<0.05)$. Except for two patients, in whom the Breslow index for the first tumour was not specified, all the others had proper registration of the tumour thickness both for the first and subsequent melanomas. Twelve out of the last patients had invasive melanoma in both the first and second melanoma; ten $(83.33 \%)$ patients developed thinner second melanoma, while thicker second melanoma was documented in two $(16.66 \%)$ patients. One of the patients, who had in situ melanoma as the first primary, developed an invasive second melanoma. All the patients with more than two melanomas developed thinner subsequent melanomas.

Ulceration and lymph node involvement were more common in the first primary tumour. Regarding stage at diagnosis, most of the subsequent melanomas were in situ or stage I, while first primary melanomas had a higher probability of being stage II or more advanced.

The median time to diagnosis was 2.75 months (25-75th percentile: 0-30.99), while the mean was 28.09 months (SD 58.511). From the subsequent melanomas, 13 (45.45\%) developed as synchronous tumours. Out of the $15(57.69 \%)$ patients with multiple lesions, four $(15.38 \%)$ had a subsequent melanoma within the first and second year. Still, five patients $(19.23 \%)$ were diagnosed with a subsequent melanoma after $>5$ years of follow-up (Fig. 1).

\section{Discussion}

The present study was conducted to document multiple primary melanomas in a large medical university centre from Romania (Cluj-Napoca), in the period from 2004 to 2020. The study focused on the number of lesions detected, tumour and patient characteristics, as well as time to subsequent diagnosis, being the first study performed in this country.

In our study comprising a series of 699 patients with melanoma, $3.71 \%$ developed a second melanoma during the follow-up, an incidence in the range reported in previous studies and similar with the one reported by Pastor-Tomás et al in Valencia, Spain (6). However, the literature presents an incidence which varies from 0.2 to $8 \%$, variability caused by the lack of homogeneity in the studies, but also by differences in ultraviolet radiation across geographical regions (6-16). Moreover, the above-reported incidence may underestimate the lifetime incidence due to limited data capture and follow-up periods (7).

Most patients in our study developed only two primary melanomas, but two patients developed four primary melanomas during follow-up. In the literature, we found a reported case of as many as 48 melanomas in one patient (17).

In $53.84 \%$ of the cases, the site of the first and second melanoma was the same, the correlation being fair. The head/neck, followed by the trunk, was the site with the highest correlation. In contrast, other studies failed to show a correlation between the first and second site $(6,18-21)$. These findings emphasise the importance of full skin examination in melanoma patients, not only at the first visit but also at follow-ups. However, close surveillance of the same body region as the initial primary melanoma in the follow-up is equally important (6-8). 
Table I. Tumour characteristics of the first and subsequent melanomas.

\begin{tabular}{|c|c|c|c|}
\hline Characteristics & First melanoma n (\%) & Subsequent melanoma n (\%) & P-value \\
\hline Histological subtype & & & 0.025 \\
\hline SSM & $16(61.5)$ & $26(78.8)$ & \\
\hline NM & $8(30.8)$ & $1(3.0)$ & \\
\hline LMM & - & $3(9.1)$ & \\
\hline ALM & - & $1(3.0)$ & \\
\hline Not specified & $2(7.7)$ & $2(6.1)$ & \\
\hline Breslow index & & & 0.001 \\
\hline In situ & $2(7.7)$ & $17(51.5)$ & \\
\hline$<1 \mathrm{~mm}$ & $3(11.5)$ & $8(24.2)$ & \\
\hline $1-2 \mathrm{~mm}$ & $6(23.1)$ & $4(12.1)$ & \\
\hline $2-4 \mathrm{~mm}$ & $10(38.5)$ & $3(9.1)$ & \\
\hline$>4 \mathrm{~mm}$ & $3(11.5)$ & $1(3.0)$ & \\
\hline Not specified & $2(7.7)$ & - & \\
\hline Ulceration & & & 0.012 \\
\hline No & $14(53.8)$ & $27(81.81)$ & \\
\hline Yes & $9(34.6)$ & $3(9.09)$ & \\
\hline Not specified & $3(11.5)$ & $3(9.09)$ & \\
\hline Lymph node involvement & & & 0.008 \\
\hline No & $19(73.1)$ & $31(93.93)$ & \\
\hline Yes & $5(19.2)$ & - & \\
\hline Not specified & $2(7.7)$ & $2(6.07)$ & \\
\hline Stage & & & 0.001 \\
\hline MIS & $2(7.7)$ & $17(51.5)$ & \\
\hline IA & $3(11.5)$ & $10(30.3)$ & \\
\hline IB & $4(15.4)$ & $4(12.1)$ & \\
\hline IIA & $3(11.5)$ & - & \\
\hline IIB & $5(19.2)$ & $2(6.1)$ & \\
\hline IIC & $1(3.8)$ & - & \\
\hline IIIA & $1(3.8)$ & - & \\
\hline IIIB & - & - & \\
\hline IIIC & $3(11.5)$ & - & \\
\hline IV & $2(7.7)$ & - & \\
\hline Not specified & $2(7.7)$ & - & \\
\hline Total & $26(100)$ & $33(100)$ & \\
\hline
\end{tabular}

SSM, superficial spreading melanoma; NM nodular melanoma; LMM, lentigo maligna melanoma; ALM, acral lentiginous melanoma.

Table II. Concordance between the site of the first and second melanoma.

\begin{tabular}{|c|c|c|c|c|c|}
\hline \multirow[b]{2}{*}{ First melanoma } & \multicolumn{5}{|c|}{ Second melanoma } \\
\hline & Head/neck (\%) & Trunk (\%) & Upper limbs (\%) & Lower limbs $(\%)$ & Total $(\%)$ \\
\hline Head/neck & $4(80)$ & $1(7.7)$ & $0(0)$ & $0(0)$ & $5(19.2)$ \\
\hline Trunk & $1(20)$ & $8(61.5)$ & $1(25)$ & $2(50)$ & $12(46.2)$ \\
\hline Upper limbs & $0(0)$ & $2(15.4)$ & $1(25)$ & $1(25)$ & $4(15.4)$ \\
\hline Lower limbs & $0(0)$ & $2(15.4)$ & $2(50)$ & $1(25)$ & $5(19.2)$ \\
\hline Total & $5(100)$ & $13(100)$ & $4(100)$ & $4(100)$ & $26(100)$ \\
\hline
\end{tabular}


Table III. Concordance between the histological subtype of the first and second melanoma.

Second melanoma

\begin{tabular}{lclllcr}
\cline { 2 - 6 } First melanoma & SSM $(\%)$ & NM $(\%)$ & LMM $(\%)$ & ALM $(\%)$ & Not specified (\%) & Total (\%) \\
\hline SSM & $15(68.2)$ & $1(100)$ & $0(0)$ & $0(0)$ & $0(0)$ & $16(61.5)$ \\
NM & $6(27.3)$ & $0(0)$ & $1(100)$ & $1(100)$ & $0(0)$ & $8(30.8)$ \\
LMM & $0(0)$ & $0(0)$ & $0(0)$ & $0(0)$ & $0(0)$ & $0(0)$ \\
ALM & $0(0)$ & $0(0)$ & $0(0)$ & $0(0)$ & $0(0)$ & $0(0)$ \\
Not specified & $1(4.5)$ & $0(0)$ & $0(0)$ & $0(0)$ & $1(100)$ & $2(7.7)$ \\
Total & $22(100)$ & $1(100)$ & $1(100)$ & $1(100)$ & $1(100)$ & $26(100)$ \\
\hline
\end{tabular}

SSM, superficial spreading melanoma; NM, nodular melanoma; LMM, lentigo maligna melanoma; ALM, acral lentiginous melanoma.

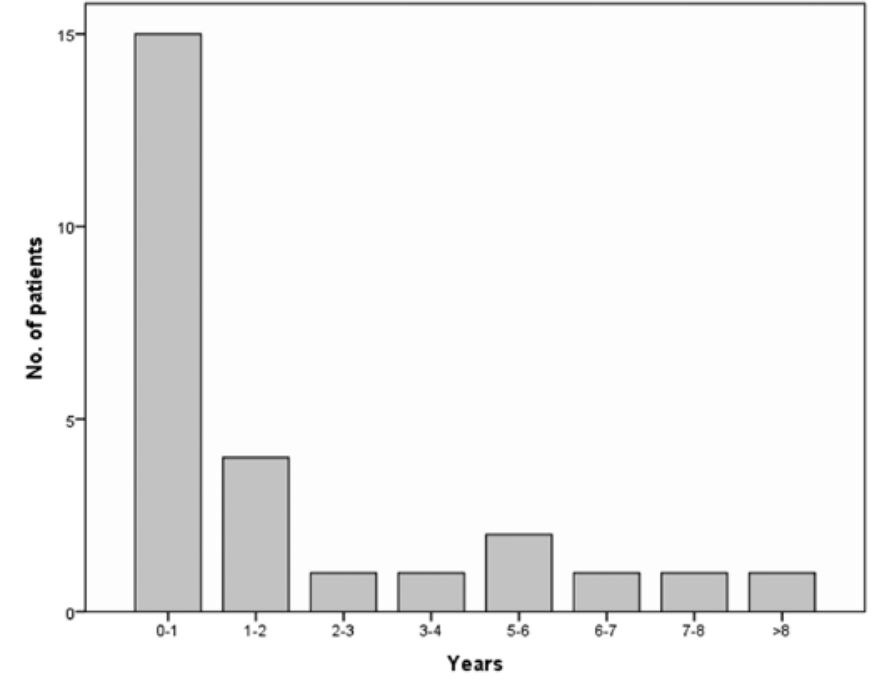

Figure 1. Time from diagnosis of the first melanoma to the diagnosis of the subsequent melanoma.

In our study, SSM and NM were, in decreasing order, the most common histopathological subtypes, in line with the results of other studies (8). While SSM was the most frequent type both in first and subsequent melanomas, NM was predominantly seen as the first melanoma. We did not find a correlation between the histological subtype of the first and second melanoma, SSM being the variant with the highest agreement.

A significant finding, reported in most previous studies, is the reduction in tumour thickness for subsequent melanomas (6-8,21-23). In our study, we observed a proportion of thin melanomas $(<1 \mathrm{~mm})$ of 19.2, 69.3, 100 and $100 \%$ for the first, second, third and fourth subsequent melanomas, respectively. This situation may be explained by early detection due to medical surveillance and self-examination. The early detection hypothesis is supported by studies that demonstrated that patients undergoing rigorous controls and adherent to regular follow-up had significantly thinner subsequent primary melanomas than those who did not $(6,7,24)$. Another possible explanation for the reduction of tumour thickness would be the potential biological difference between the first and second melanoma (6). Multiple studies have suggested that melanoma is in fact, not a single entity but a group of different neoplasms with variable etiopathogenesis, biologic behaviour and prognosis (25-30). This hypothesis needs to be tested in further studies in patients with multiple melanomas.

Among patients with multiple primary melanomas, synchronous lesions were reported in $26-40 \%$ of the cases, while the remainder develops as asynchronous tumours (7). In our study, $45.45 \%$ of the lesions presented as synchronous lesions, underscored the importance of total body examination during the first visit in melanoma patients. Previous studies have shown that the risk of a subsequent primary melanoma is highest in the first year of the incident primary melanoma and remains higher in the first five years (6-8). However, cases reported up to 2 to 3 decades after the first melanoma and some studies indicate that the risk remains stable in time (6). In our study, almost $20 \%$ of the patients developed a second melanoma after more than five years of follow-up. Moreover, a patient who presented with a very thin first melanoma (BI=0.25 mm), developed two synchronous in situ melanomas after seven years of follow-up. All these data reveal the importance of lifetime clinical follow-up in melanoma patients, although some guidelines support a limited follow-up of one year for stage IA melanoma (31).

The main strength of our study is the homogeneous information collection in a single institution for a long period. The main limitation consists in the relatively low number of cases, but this can be explained by the fact that data was collected in a single centre.

In conclusion, almost $4 \%$ of melanoma patients in our centre developed subsequent melanomas during 2004 and 2020. Most patients developed only two primary melanomas, and we could not find a strong correlation between the sites and the histopathological subtypes of the first and subsequent melanoma. The most important findings of our study are the reduction in tumour thickness in subsequent melanomas as well as the possibility of diagnosing a subsequent melanoma more than five years after the first diagnosis. However, the risk is highest in the first year. Our findings highlight the need for lifetime clinical follow-up and self-examination in melanoma patients regardless of the first melanoma stage. 


\section{Acknowledgements}

Not applicable.

\section{Funding}

The present study was partially supported by the grant PN-III-P1-1.2-PCCDI-2017-0341.

\section{Availability of data and materials}

The datasets used and/or analysed during the current study are available from the corresponding author on reasonable request.

\section{Authors' contributions}

LU and SS contributed to the research-creation and design of the study, data acquisition, analysis and interpretation of data, statistical analysis, manuscript drafting, and critical revision of the manuscript for valuable intellectual content. IZ contributed to the data acquisition, analysis and interpretation of data, statistical analysis, manuscript drafting, and critical review of the manuscript for valuable intellectual content. IC and AV contributed to to the research-creation and design of the study, data acquisition, and critical revision of the manuscript for valuable intellectual content. SV performed the analysis and interpretation of data, and the critical review of the manuscript for valuable intellectual content. RC contributed to the research-creation and design, and critical revision of the manuscript for valuable intellectual content. All authors read and approved the manuscript.

\section{Ethics approval and consent to participate}

The present study was approved by the Ethics Committee of the 'Iuliu Hatieganu' University of Medicine and Pharmacy (Cluj-Napoca, Romania). All the participants gave their consent to be included in the study.

\section{Patient consent for publication}

Not applicable.

\section{Competing interests}

The authors declare that they have no competing interests.

\section{References}

1. Jemal A, Bray F, Center MM, Ferlay J, Ward E and Forman D: Global cancer statistics. CA Cancer J Clin 61: 69-90, 2011.

2. Godar DE: Worldwide increasing incidence of cutaneous malignant melanoma. J Skin Cancer 2011: 858425, 2011

3. Tripp MK, Watson M, Balk SJ, Swetter SM and Gershenwald JE: State of the science on prevention and screening to reduce melanoma incidence and mortality: The time is now. CA Cancer J Clin 66: 460-480, 2016.

4. Ribas A, Hamid O, Daud A, Hodi FS, Wolchok JD, Kefford R, Joshua AM, Patnaik A, Hwu WJ, Weber JS, et al: Association of pembrolizumab with tumor response and survival among patients with advanced melanoma. JAMA 315: 1600-1609, 2016.

5. Long GV, Stroyakovskiy D, Gogas H, Levchenko E, de Braud F Larkin J, Garbe C, Jouary T, Hauschild A, Grob JJ, et al: Dabrafenib and trametinib versus dabrafenib and placebo for Val600 BRAF-mutant melanoma: A multicentre, double-blind, phase 3 randomised controlled trial. Lancet 386: 444-451, 2015.
6. Pastor-Tomás N, Martínez-Franco A, Bañuls J, Peñalver JC Traves V, García-Casado Z, Requena C, Kumar R and Nagore E: Risk factors for the development of a second melanoma in patients with cutaneous melanoma. J Eur Acad Dermatol Venereol: Mar 12,2020 (Online ahead of print).

7. Adler NR, Kelly JW, Haydon A, McLean CA and Mar VJ: Clinicopathological characteristics and prognosis of patients with multiple primary melanomas. Br J Dermatol 178: e44-e45, 2018.

8. Claeson M, Holmström P, Hallberg S, Gillstedt M, Gonzalez H, Wennberg AM and Paoli J: Multiple primary melanomas: A common occurrence in Western Sweden. Acta Derm Venereol 97: 715-719, 2017.

9. van der Leest RJT, Flohil SC, Arends LR, de Vries E and Nijsten T: Risk of subsequent cutaneous malignancy in patients with prior melanoma: A systematic review and meta-analysis. J Eur Acad Dermatology Venereol 29: 1053-1062, 2015.

10. Caini S, Boniol M, Botteri E, Tosti G, Bazolli B, Russell-Edu W, Giusti F, Testori A and Gandini S: The risk of developing a second primary cancer in melanoma patients: A comprehensive review of the literature and meta-analysis. J Dermatol Sci 75: 3-9, 2014.

11. Bradford PT, Freedman DM, Goldstein AM and Tucker MA: Increased risk of second primary cancers after a diagnosis of melanoma. Arch Dermatol 146: 265-272, 2010.

12. Spanogle JP, Clarke CA, Aroner S and Swetter SM: Risk of second primary malignancies following cutaneous melanoma diagnosis: A population-based study. J Am Acad Dermatol 62: 757-767, 2010.

13. Crocetti E, Guzzinati S, Paci E, Falcini F, Zanetti R, Vercelli M, Rashid I, De Lisi V, Russo A, Vitarelli S, et al: The risk of developing a second, different, cancer among 14560 survivors of malignant cutaneous melanoma: A study by AIRTUM (the Italian Network of Cancer Registries). Melanoma Res 18: 230-234, 2008.

14. Bhatia S, Estrada-Batres L, Maryon T, Bogue M and Chu D: Second primary tumors in patients with cutaneous malignant melanoma. Cancer 86: 2014-2020, 1999.

15. Espinosa P, Pfeiffer RM, García-Casado Z, Requena C, Landi MT, Kumar R and Nagore E: Risk factors for keratinocyte skin cancer in patients diagnosed with melanoma, a large retrospective study. Eur J Cancer 53: 115-124, 2016.

16. Moore MM, Geller AC, Warton EM, Schwalbe J and Asgari MM: Multiple primary melanomas among 16,570 patients with melanoma diagnosed at Kaiser Permanente Northern California, 1996 to 2011. J Am Acad Dermatol 73: 630-636, 2015.

17. Slingluff CL Jr, Vollmer RT and Seigler HF: Multiple primary melanoma: Incidence and risk factors in 283 patients. Surgery 113: 330-339, 1993.

18. Stam-Posthuma JJ, van Duinen C, Scheffer E, Vink J and Bergman W: Multiple primary melanomas. J Am Acad Dermatol 44: 22-27, 2011.

19. Titus-Ernstoff L, Perry AE, Spencer SK, Gibson J, Ding J, Cole B and Ernstoff MS: Multiple primary melanoma: Two-year results from a population-based study. Arch Dermatol 142: 433-438, 2006.

20. Siskind V, Hughes MC, Palmer JM, Symmons JM, Aitken JF, Martin NG, Hayward NK and Whiteman DC: Nevi, family history, and fair skin increase the risk of second primary melanoma. J Invest Dermatol 131: 461-467, 2011.

21. Johnson TM, Hamilton T and Lowe L: Multiple primary melanomas. J Am Acad Dermatol 39: 422-427, 1998.

22. Vecchiato A, Pasquali S, Menin C, Montesco MC, Alaibac M, Mocellin S, Campana LG, Nitti D and Rossi CR: Histopathological characteristics of subsequent melanomas in patients with multiple primary melanomas. J Eur Acad Dermatol Venereol 28: 58-64, 2014

23. DiFronzo LA, Wanek LA and Morton DL: Earlier diagnosis of second primary melanoma confirms the benefits of patient education and routine postoperative follow-up. Cancer 91: 1520-1524, 2001.

24. De Giorgi V, Rossari S, Papi F, Gori A, Alfaioli B, Grazzini M, Crocetti E, Verdelli A, Foo CW and Lotti T: Multiple primary melanoma: The impact of atypical naevi and follow-up. Br J Dermatol 163: 1319-1322, 2010.

25. Ilie MA, Caruntu C, Lupu M, Lixandru D, Tampa M, Georgescu SR, Bastian A, Constantin C, Neagu M, Zurac SA and Boda D: Current and future applications of confocal laser scanning microscopy imaging in skin oncology. Oncol Lett 17: 4102-4111, 2019. 
26. Ancuceanu R, Dinu M, Neaga I, Laszlo FG and Boda D Development of QSAR machine learning-based models to forecast the effect of substances on malignant melanoma cells. Oncol Lett 17: 4188-4196, 2019.

27. Caruntu C, Boda D, Constantin C, Caruntu A and Neagu M: Catecholamines increase in vitro proliferation of murine B16F10 melanoma cells. Acta Endocrinol 10: 545-558, 2014.

28. Boda D: Cellomics as integrative omics for cancer. Curr Proteomics 10: 237-245, 2013.

29. Stefan O, Tudor G, Constantinescu C, Luca C, Boda D, Caruntu C, Cioplea M, Nichita L and Zurac SA: E-cadherin and N-cadherin expression pattern in common melanocytic nevi. Virchows Archiv 475 (Suppl): S28, 2019.
30. Zurac S, Neagu M, Constantin C, Cioplea M, Nedelcu R, Bastian A, Popp C, Nichita L, Andrei R, Tebeica T, et al: Variations in the expression of TIMP1, TIMP2 and TIMP3 in cutaneous melanoma with regression and their possible function as prognostic predictors. Oncol Lett 11: 3354-3360, 2016.

31. Garbe C, Amaral T, Peris K, Hauschild A, Arenberger P, Bastholt L, Bataille V, Del Marmol V, Dréno B, Fargnoli MC, et al: European consensus-based interdisciplinary guideline for melanoma. Part 1: Diagnostics-update 2019. Eur J Cancer 126: $141-158,2020$.

c) (1) (2) This work is licensed under a Creative Commons EY NG No Attribution-NonCommercial-NoDerivatives 4.0 International (CC BY-NC-ND 4.0) License. 